\title{
Teor de arsênio e adsorção competitiva arsênio/fosfato e arsênio/sulfato em solos de Minas Gerais, Brasil
}

\author{
Arsenate content, and arsenate/phosphate and arsenate/sulphate competitive adsorption in soils \\ from Minas Gerais, Brazil.
}

\author{
Mari Lucia Campos ${ }^{{ }^{*}}$ Luiz Roberto Guimarães Guilherme ${ }^{\text {II }}$ Alessandra Silveira Antunes ${ }^{\text {II }}$ \\ Karine Sousa Carsten Borges ${ }^{I}$
}

\section{RESUMO}

A alta toxicidade de As para homens e animais gera a necessidade de estudos do comportamento químico do arsenato nos solos que possam auxiliar na mitigação de áreas contaminadas com arsênio. Este trabalho teve por objetivo avaliar o teor total e a adsorção de As na ausência e presença dos ânions fosfato e sulfato em seis diferentes classes de solos do estado de Minas Gerais, Brasil. Os solos alvo deste estudo são: o Neossolo Flúvico (RU), Gleissolo Háplico (GX), Gleissolo Melânico (GM), Latossolo Vermelho Distrófico (LVd), coletados em Lavras; Neossolo Quartzarênico (RQ), coletado em Itutinga e o Latossolo Amarelo Distrófico (LAd), coletado em Rosário, no estado de Minas Gerais. As amostras de solo foram secas, moídas e peneiradas em peneira de $2,0 \mathrm{~mm}$ para execução do teste de adsorção e peneiradas em peneira plástica com malha de $1,5 \mathrm{~mm}$ para determinação do teor de As, o qual foi determinado pelo método 3051A. A adsorção de As foi avaliada na dose de1500 $\mu \mathrm{mol} \mathrm{L} L^{-1}$ de As, $1500 \mu \mathrm{mol} \mathrm{L} L^{-1}$ de As $+1500 \mu \mathrm{mol} \mathrm{L} L^{-1}$ de Pe $1500 \mu \mathrm{mol} L^{-1}$ de $A s+750 \mu \mathrm{molL}^{-1}$ de $S$, em relação solo:solução final de 1:100, a pH 5,5 e força iônica de $15 \mathrm{mmol} \mathrm{L}^{-1}$. Os seis solos apresentaram teor médio de As entre 0,14 e 9,3mgkg-1. A porcentagem adsorvida de arsênio na ausência dos outros ânions seguiu a sequência $G M>L V d=R U=L A d=G X=R Q$. A adição de fosfato e sulfato reduziu a porcentagem de arsênio adsorvido $e$, por consequência, houve um aumentou na concentração de arsênio disponível na solução do solo.

Palavras-chave: contaminação química, elementos-traço, sorção.

\section{ABSTRACT}

The high toxicity of arsenic to humans and animals creates the need to study the chemical behavior of arsenate in soils that can help in the mitigation of areas contaminated with arsenic. This work aimed to evaluate the total content and adsorption in the absence and presence of phosphate and sulfate anions in six different soil classes in the state of Minas Gerais, Brazil. Soils aim of this study are: Fluvic Neosol (RU), Haplic Gleysol (GX), Melanic Gleysol (GM) and Typical Distrophic Red Latosol (LVd) collected in Lavras, MG; Quartzenic Neosol (RQ) collected in Itutinga, MG, and Typical Distrophic Yellow Latosol (Lad) collected in Rosário, MG. Soil samples were dried, ground and sieved through a sieve of $2 \mathrm{~mm}$ for the test run of adsorption and sieved through a sieve with a mesh of plastic $1.5 \mathrm{~mm}$ for the As determination. The As content was determined by the method $3051 \mathrm{~A}$. The rate $1500 \mu \mathrm{molL} \mathrm{L}^{-1} \mathrm{As}, 1500 \mu \mathrm{mol} \mathrm{L} \mathrm{L}^{-1} \mathrm{As}+1500 \mu \mathrm{mol} \mathrm{L} \mathrm{L}^{-1}$ $P, 1500 \mu \mathrm{mol} \mathrm{L} L^{-1}$ As $+750 \mu \mathrm{mol} \mathrm{L} L^{-1}$ As were used to evaluate As adsorption, soil solution rate of 1:100, pH 5,5 and ionic force of $15 \mathrm{mmol} . \mathrm{L}^{-1}$. The mean As content in the six soils was between 0,14 and $9,3 \mathrm{mgkg}^{-1}$. The adsorpted percentage of As in absence of other anions followed the sequence $G M>L V d=R U=L A d=G X=R Q$. Phosphate and sulphate addition reduced the percentage of adsorpted arsenic, consequently there was an increase of disponible arsenic in the soil solution.

Key words: chemical contamination, sorption, trace elements.

\section{INTRODUÇÃO}

O arsênio (As) é elemento-traço altamente tóxico ao homem, animais e medianamente tóxico às plantas. A maioria dos humanos é exposta a baixos níveis de As, principalmente através de ingestão de alimentos, água e inalação de arsênio do ar (BASU et al., 2001). Entretanto, a exposição a arsênio via ingestão de produtos agrícolas e de solo resulta em significante risco à saúde (ZAKHAROVA et al., 2002).

$\mathrm{O}$ arsênio no solo pode ser originário de fontes naturais como intemperismo de rochas,

'Departamento de Solos e Recursos Naturais (DSRN), Centro de Ciências Agroveterinárias (CAV), Universidade do Estado de Santa Catarina (UDESC), Av. Luiz de Camões, 2090, 88520-000, Lages, SC, Brasil. E-mail: mari.lucia03@gmail.com. *Autor para correspondência.

IIDepartamento de Ciência do Solo, Universidade Federal de Lavras (UFLA), Lavras, MG, Brasil. 
a atividade vulcânica, aerossóis de água do mar e volatilização microbiana (NRIAGU \& PACYNA, 1988), e de fontes antropogênicas como pesticidas, herbicidas, fertilizantes, emitido durante a mineração e fundição do ouro, chumbo, cobre e níquel, ferro e combustão de carvão (SPARKS, 1995; SMITH et al., 1998; BAIRD, 2002).

No Brasil, os primeiros registros de análise da concentração de arsênio no solo foram efetuados por CURI \& FRANZMEIER (1987). Esses autores encontraram teores de As em Latossolo Vermelho distroférrico variando de 6 a $10 \mathrm{mg} \mathrm{kg}^{-1}$. Teor médio total de As de 5,92 $\mathrm{mg} \mathrm{kg}^{-1}$ foi observado em 15 Latossolos localizados em áreas experimentais das Embrapas e dois Latossolos do campus da UFLA, MG (CAMPOS et al., 2007). Entretanto, há relatos de ocorrência de contaminação de águas (até $0,36 \mathrm{mg} \mathrm{L}^{-1}$ ), solos (até $860 \mathrm{mg} \mathrm{kg}^{-1}$ ) e sedimentos (até $3.200 \mathrm{mg} \mathrm{kg}^{-1}$ ) por As nas proximidades de áreas industriais ou de mineração no quadrilátero ferrífero, MG (MAGALHÃES \& PFEIFFER, 1995; MATSCHULLAT, 2000; MAGALHÃES et al., 2001). No solo, o As é adsorvido fracamente na matéria orgânica e em argilominerais, e, à semelhança do fósforo, é altamente adsorvido a óxidos, especialmente em valor de $\mathrm{pH}$ baixo (McBRIDE, 1994). A capacidade de adsorção de As pelos solos fornece indícios do poder-tampão do sistema, visto que muitos atributos do solo (como textura, teor de óxidos de $\mathrm{Fe}$ e $\mathrm{Al}$, entre outros) interferem na capacidade-tampão e, por consequência, influenciam na perda por lixiviação.

O manejo de solos contaminados com arsênio passa primeiramente pelo entendimento do comportamento químico desse elemento no solo. No caso do arsênio, a disponibilidade, para absorção pelas plantas e para lixiviação, é aumentada pela adição de fertilizantes que contenham oxiânions, como o fosfato e o sulfato. O aumento do As disponível após adição de oxiânions ao solo está relacionado à capacidade do fosfato e do sulfato em competir com o arsenato pelos sítios de adsorção presentes na superfície dos minerais do solo (MELAMED et al., 1995; GEELHOED et al., 1997; GUSTAFSSON, 2001).

A alta toxicidade de As para homens e animais, aliado à pequena disponibilidade de informação sobre comportamento do arsênio em solos tropicais gera a necessidade de estudos do comportamento químico do arsenato nesses solos que possam auxiliar na mitigação de áreas contaminadas com arsênio. Este trabalho teve por objetivo avaliar o teor total e a adsorção de As na ausência e presença dos ânions fosfato e sulfato em seis diferentes classes de solos do estado de Minas Gerais, Brasil.

\section{MATERIAL E MÉTODO}

Foram utilizadas amostras da camada superficial $(0-20 \mathrm{~cm})$ de seis classes de solos coletados no sul de Minas Gerais (Tabela 1): o Neossolo Flúvico (RU) (coordenadas UTM x: 503.662 e y: 7.646.370), Gleissolo Háplico (GX), Gleissolo Melânico (GM), Latossolo Vermelho Distrófico (LVd) (coordenadas UTM: x: 0503646 e y: 7652507 - 23K), coletados no município de Lavras; Neossolo Quartzarênico (RQ), coletado no município de Itutinga (coordenadas UTM: x: 0540201 e y: 7644073-23K) e o Latossolo Amarelo Distrófico (LAd), coletado no município de Rosário. Todas as amostras de solos foram coletadas em locais reconhecidamente não sujeitos à contaminação intencional com As (as únicas fontes possíveis são deposição atmosférica e adição de insumos que, porventura, contenham As) e preferencialmente em áreas com vegetação nativa. Essas classes de solos foram escolhidas por possuírem diferentes texturas e teor de matéria orgânica. A caracterização físicoquímica e mineralógica desses solos foi realizada por SOUZA et al. (2006) e FERNANDES et al. (1999) (Tabela 1). Todas as análises foram conduzidas nos laboratórios do Departamento de Ciência do Solo da Universidade Federal de Lavras (UFLA). Para determinação do teor de As, as amostras foram inicialmente peneiradas em peneira plástica com malha de $1,5 \mathrm{~mm}$ de abertura. Após isso, elas foram trituradas até passar inteiramente por uma peneira descartável de náilon com malha de $0,15 \mathrm{~mm}$ de abertura. A extração de As seguiu o protocolo do método 3051A da USEPA (USEPA, 1995), que prevê a digestão de 1,0 a $0,5 \mathrm{~g}$ de material em $10 \mathrm{~mL}$ de $\mathrm{HNO}_{3}$ concentrado em forno de microondas com tubos de Teflon $^{\circledR}$ PTFE (politetrafluoroetileno), à pressão de $0,76 \mathrm{MPa}$ por $10 \mathrm{~min}$. As amostras de solo e das amostras controle (AC02-115 e AC 02-103) foram analisadas em triplicata. As amostras controle, denominadas AC 115 e AC 103, são provenientes de um programa inter laboratorial de análises de solos, o North American Proficiency Testing (NAPT -http:// www.naptprogram.org/), coordenado pela Soil Science Society of America.

Para a realização dos testes de adsorção e competição, foram preparadas suspensões com terra fina seca ao ar (TFSA) dos seis solos em solução salina de $\mathrm{NaCl} 15 \mathrm{mmolL}^{-1}$ e pH ajustado a 5,5 $\pm 0,2$. $\mathrm{O}$ ajuste do valor de $\mathrm{pH}$ foi realizado com $\mathrm{NaOH}$ e $\mathrm{HCl} 10 \mathrm{mmol} \mathrm{L}^{-1}$. A relação solo:solução final foi de 1:100, e todas as amostras foram avaliadas em triplicata. Após a estabilização do $\mathrm{pH}$, as suspensões foram colocadas para reagir com $1500 \mu \mathrm{mol} \mathrm{L}^{-1}$ de As, 
Tabela 1 - Características químicas, físicas e mineralógicas da camada 0-20 cm dos solos LAd, LVd, RU, GX, GM e RQ, determinadas por FERNANDES et al. (1999) e SOUZA et al. (2006). Teor médio de arsênio nos seis solos e nas amostras controle (AC), limite de detecção qualitativo do método USEPA 3051A e porcentagem média de adsorção As.

\begin{tabular}{|c|c|c|c|c|c|c|c|c|c|c|c|c|c|}
\hline \multirow{2}{*}{ Materiais } & \multicolumn{4}{|c|}{----Textura---- ---Mineralogia--- } & \multicolumn{3}{|c|}{--------Ataque sulfúrico------- } & \multicolumn{2}{|c|}{--------Fe------- } & \multirow{2}{*}{ MO } & \multirow{2}{*}{ CTC } & \multicolumn{2}{|c|}{ 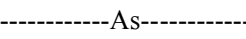 } \\
\hline & Arg & Are & $\mathrm{Ct}$ & $\mathrm{Gb}$ & $\mathrm{SiO}_{2}$ & $\mathrm{Al}_{2} \mathrm{O}_{3}$ & $\mathrm{Fe}_{2} \mathrm{O}_{3}$ & DCB & $\mathrm{Ox}$ & & & Teor & Ads \\
\hline & ------- & ---- & $-\cdots$ & ------ & ------ & $\mathrm{kg}^{-1}---$ & ------- & ------ & ----- & -------- & $\mathrm{mmol}_{\mathrm{c}} \mathrm{kg}^{-1}$ & $\mathrm{Mg} \mathrm{kg}^{-1}$ & $\%$ \\
\hline Lad & 220 & 680 & 580 & 220 & 80 & 33 & 11 & 3,6 & 0,1 & 14 & 48 & $0,33 \pm 0,3$ & $58,6 \mathrm{~b}$ \\
\hline LVd & 690 & 250 & 190 & 484 & 159 & 270 & 125 & 46 & 2,2 & 33 & 60 & $0,14 \pm 0,1$ & $57,7 \mathrm{~b}$ \\
\hline RU & 310 & 420 & 50 & 17 & 209 & 221 & 75 & 4,3 & 0,1 & 41 & 86 & $1,09 \pm 0,3$ & $57,2 \mathrm{~b}$ \\
\hline GX & 160 & 500 & 12 & 3,4 & 84 & 112 & 13 & 0,3 & 0,1 & 35 & 72 & $0,43 \pm 0,4$ & $58,4 \mathrm{~b}$ \\
\hline GM & 370 & 240 & 18 & 20 & 126 & 176 & 13 & 0,9 & 0,2 & 245 & 150 & $9,26 \pm 1,0$ & $65,9 \mathrm{a}$ \\
\hline RQ & 60 & 920 & 681 & 220 & 31 & 36 & 11 & 2,0 & 0,1 & 14 & 43 & $0,86 \pm 0,3$ & $55,0 \mathrm{c}$ \\
\hline AC $02-115$ & - & - & - & - & - & - & - & - & - & - & - & $1,44 \pm 0,1$ & - \\
\hline AC $02-103$ & - & - & - & - & - & - & - & - & - & - & - & $2,09 \pm 0,5$ & - \\
\hline $\mathrm{LDQ}^{*}$ & - & - & - & - & - & - & - & - & - & - & - & 0,06 & - \\
\hline
\end{tabular}

Arg: argila; Are: areia; Ct: caulinita da fração argila; Gb: gibbsita da fração argila; $\mathrm{DCB}: \mathrm{Fe}_{2} \mathrm{O}_{3}$ extraído pelo ditionito citrato-bicarbonato; Ox: $\mathrm{Fe}_{2} \mathrm{O}_{3}$ extraído pelo oxalato ácido de amônio.

As letras minúsculas comparam a \% de adsorção média entre solos, sendo que as médias seguidas de mesma letra, na coluna, não diferem entre si pelo teste de Scott-Knott a 5\% de probabilidade.

AC: amostras-controle denominadas AC 115 e AC 103, que são provenientes de um programa interlaboratorial de análises de solos, o North American Proficiency Testing (NAPT - http://www.naptprogram.org/), coordenado pela Soil Science Society of America.

$* L D Q=F d *(\mathrm{M} \pm k * s)$

(AMERICAN PUBLIC HEALTH ASSOCIATION, 1989), em que LDQ é o limite de detecção qualitativo do método analítico, Fd é o fator de diluição das amostras, M a média das provas em branco, k o coeficiente de Kaiser que possui valor fixo igual a três (SKOOG, 2002) e s é o desvio padrão das provas em branco.

$1500 \mu \mathrm{mol} \mathrm{L}-1$ de As $+1500 \mu \mathrm{mol} \mathrm{L}{ }^{-1}$ de P e $1500 \mu \mathrm{mol}$ $\mathrm{L}^{-1}$ de $\mathrm{As}+750 \mu \mathrm{mol} \mathrm{L} \mathrm{L}^{-1} \mathrm{~S}$. Para confecção das soluções-padrão, foram utilizados $\mathrm{Na}_{2} \mathrm{HAsO}_{4} \cdot 7 \mathrm{H}_{2} \mathrm{O}$, $\mathrm{Na}_{2} \mathrm{HPO}_{4} \cdot 7 \mathrm{H}_{2} \mathrm{O}$ e $\mathrm{Na}_{2} \mathrm{SO}_{4}$. As condições para condução do estudo de adsorção e competição foram definidas após avaliação das espécies em solução, para tanto foi utilizado o software MINTEq. Essa avaliação garantiu que a $\mathrm{pH} 5,5$, FI $15 \mathrm{mmol} \mathrm{L}^{-1}, 95 \%$ do As, $\mathrm{P}$ e $\mathrm{S}$ adicionados estejam nas formas $\mathrm{H}_{2} \mathrm{AsO}_{4}^{-}, \mathrm{H}_{2} \mathrm{PO}_{4}{ }^{-} \mathrm{e}$ $\mathrm{SO}_{4}{ }^{-2}$, respectivamente. $\mathrm{O}$ delineamento utilizado foi o inteiramente casualizado com três repetições.

O tempo de contato entre o solo e a solução foi de $72 \mathrm{~h}$, divididas em ciclos de $12 \mathrm{~h}$ de agitação e $12 \mathrm{~h}$ em repouso. Após as $72 \mathrm{~h}$ de reação, a suspensão de cada solo foi centrifugada e o sobrenadante retirado para leitura.

A quantidade adsorvida foi calculada, aplicando-se as seguintes equações: Asads $=\{(\mathrm{Ci}$ $\left.\mathrm{Ce})\left[\left(\mathrm{V}_{1}+\mathrm{V}_{2}\right) / \mathrm{Ms}\right]\right\} ; \quad$ Asadc $=\left\{\left(\mathrm{Ci}\left[\left(\mathrm{V}_{1}+\mathrm{V}_{2}\right) / \mathrm{Ms}\right]\right\} \quad \mathrm{e}\right.$ $\%$ As $=($ Asads/Asadc)*100 (CAMPOS et al., 2006; SOARES \& CASAGRANDE, 2009). Em que Asads é o arsênio adsorvido, em $\mathrm{mg} \mathrm{kg}^{-1}$ de solo; Ce é a concentração de equilíbrio depois de $72 \mathrm{~h}$; Ms é a massa do solo, em g; Asadc é o arsênio adicionado, em mg L ${ }^{-1}$ de solo; $\mathrm{Ci}$ é a concentração inicial de
As, em $\mathrm{mgL}^{-1} ; \mathrm{V}_{1}$ é o volume, em $\mathrm{mL}$, da solução padrão de arsênio fosfato e sulfato; $\mathrm{V}_{2}$ é o volume, em $\mathrm{mL}$, de solução de fundo $\left(\mathrm{NaCl} 15 \mathrm{mmol} \mathrm{L}^{-1}\right)$; e \%As é a porcentagem de arsênio adsorvido pelo solo. Além do cálculo das quantidades de As adsorvidas, foi determinado também o coeficiente de distribuição de As $\left(\mathrm{Kd}\right.$, em $\left.\mathrm{L} \mathrm{kg}^{-1}\right)$, nas diferentes amostras pela equação $\mathrm{Kd}=$ Asads/Ce (TAN, 1992), porém, para melhor visualização dos valores de $\mathrm{Kd}$, esses foram apresentados em base logarítmica.

A quantificação do teor de As, em todas as análises, foi realizada por espectrofotometria de absorção atômica, usando-se equipamento Perkin Elmer AAnalyst 800, com atomização eletrotérmica em forno de grafite. As condições de leitura utilizadas foram temperatura de pirólise de $500^{\circ} \mathrm{C}$ (recomendação do fabricante) e $0,003 \mathrm{mg}$ de $\mathrm{Mg}$ na forma de $\mathrm{Mg}\left(\mathrm{NO}_{3}\right)_{2}$, como modificador químico (NIEDZIELSKI et al., 2002). A análise de variância e correlação de Pearson entre os atributos do solo e a porcentagem adsorvida foi feita, utilizando-se o software SISVAR 4.3 (FERREIRA, 2000). Para comparação entre médias, utilizou-se o teste de ScottKnott a 5\%.

Ciência Rural, v.43, n.6, jun, 2013. 


\section{RESULTADOS E DISCUSSÃO}

Os seis solos estudados apresentaram teores naturais entre $0,14 \pm 0,1 \mathrm{mg} \mathrm{kg}^{-1}(\mathrm{GX})$ e $9,3 \pm 1,0 \mathrm{mg} \mathrm{kg}^{-1}$ (LVd) (Tabela 1). Com exceção do solo $\mathrm{LVd}$, todos os outros apresentaram teor de As abaixo do valor mínimo apresentado por WENZEL et al. (2002), que considera como concentração normal de As em solo o intervalo $10-120 \mathrm{mg} \mathrm{kg}^{-1}$. Os solos LAd, RU, RQ, GM e GX apresentaram teores de As menores que os relatados por CURI \& FRANZMEIER (1987) para Latossolo Roxo (6 a 10 $\mathrm{mg} \mathrm{kg}^{-1}$ ) e Latossolo Ferrífero (36mg kg-1) no Brasil. A concentração de As em solos ditos não contaminados encontra-se abaixo de $10 \mathrm{mg} \mathrm{kg}^{-1}$ (FITZ \& WENZEL, 2002; SMEDLEY \& KINNIBURGH, 2002), sendo que concentrações mais elevadas devem-se a fontes tanto naturais quanto antropogênicas (FITZ \& WENZEL, 2002).São fontes naturais o intemperismo de rochas, a atividade vulcânica, aerossóis de água do mar e volatilização microbiana (NRIAGU \& PACYNA, 1988) e fontes antropogênicas o uso de pesticidas, herbicidas, fertilizantes, emitido durante a mineração e fundição do ouro, chumbo, cobre e níquel, produção de ferro e aço, combustão de carvão (SPARKS, 1995; SMITH et al., 1998; BAIRD, 2002) e irrigação com água contaminada (ROYCHOWDHURY et al., 2002).

A porcentagem de adsorção de As para os solos estudados variou entre $55 \%$ e $66 \%$ (Tabela 1), sendo que o solo GM foi o que apresentou maior porcentagem de adsorção. A maior capacidade de adsorção de GM pode estar relacionada à presença de formas amorfas de óxidos de ferro, devido à exposição frequente desse solo a ciclos de inundação e drenagem. Óxidos de ferro pobremente cristalinos possuem uma forte afinidade de adsorção por arsênio (RAVEN et al., 1998; JAIN et al., 1999), sendo que o arsenato forma complexos mais fortes com a ferridrita que com a gibbsita ou alofana (GUSTAFSSON, 2001). No presente trabalho, a relação $\mathrm{Fe}_{\mathrm{ox}} / \mathrm{Fe}_{\mathrm{DCB}}$ correlacionouse significativamente com a porcentagem adsorvida de arsênio (Tabela 2), concordando com o observado por GUSTAFSSON (2001).O mecanismo de adsorção do arsenato é de formação de esfera interna (adsorção especifica) (GUSTAFSSON, 1995; SMITH et al., 1999), sendo que nos óxidos ocorre ligação do tipo binuclear ou bidentada (HIEMSTRA \& RIEMSDIJK, 1999). Os atributos argila, $\mathrm{Al}_{2} \mathrm{O}_{3}$ e $\mathrm{Fe}_{2} \mathrm{O}_{3}$ obtidos por ataque sulfúrico não apresentaram correlação significativa com porcentagem adsorção de arsênio neste estudo.

A avaliação da adsorção competitiva revelou que a presença do sulfato e fosfato resultou em diminuição da adsorção do arsenato, quando
Tabela 2 - Coeficiente de correlação de Pearson entre os atributos químicos de seis solos e porcentagem de adsorção de arsênio na dose inicial $1500 \mu \mathrm{mol} \mathrm{L}{ }^{-1}$.

\begin{tabular}{lc}
\hline Atributo & Correlação \\
\hline Argila & $0,32^{\mathrm{ns}}$ \\
$\mathrm{Al}_{2} \mathrm{O}_{3}$ & $0,38^{\mathrm{ns}}$ \\
$\mathrm{Fe}_{2} \mathrm{O}_{3}$ & $0,17^{\mathrm{ns}}$ \\
$\mathrm{Fe}_{\mathrm{ox}} / \mathrm{Fe}_{\mathrm{DCB}}$ & $0,46^{*}$ \\
\hline
\end{tabular}

*Significativo a $1 \%$, * Significativo a $5 \%$, ${ }^{\text {ns }}$ Não significativo. $\mathrm{Al}_{2} \mathrm{O}_{3}$ e $\mathrm{Fe}_{2} \mathrm{O}_{3}$ obtido por ataque sulfúrico e Feox/FeDCB: DCB: $\mathrm{Fe}_{2} \mathrm{O}_{3}$ extraído pelo ditionito citrato-bicarbonato; Ox: $\mathrm{Fe}_{2} \mathrm{O}_{3}$ extraído pelo oxalato ácido de amônio.

comparado com adsorção na ausência desses ânions (Figura 1). O sulfato e o fosfato apresentaram capacidade de competição semelhante nos solos LAd, LVd, GX e RU. No caso dos solos RQ e GM, a adsorção de arsenato foi maior na presença do sulfato que na presença do fosfato (Figura 1), sendo que, para o solo GM, a adsorção de As foi de $65 \%$ na ausência do fosfato e caiu para $21 \%$ na presença desse oxiânion. Esse comportamento esta relacionado à similaridade química entre esses dois ânions (e.g., valores semelhantes de pKa), o que confere semelhante capacidade de competição pelos sítios de adsorção dos coloides do solo (MELAMED et al., 1995). Além disso, arsenato e fosfato possuem capacidade de formar complexo de esfera interna (GEELHOED et al., 1997; GUSTAFSSON, 2001), enquanto que o sulfato, em solos de cargas variáveis, reage preferencialmente em superfícies carregadas positivamente (CHARLET et al., 1993).

Outro indicativo do efeito de competição é a diminuição nos valores de $\mathrm{Kd}$ para As, quando, na presença de sulfato e/ou fosfato (Figura 1). Para os solos estudados, a diminuição do Kd é mais intensa na presença do ânion fosfato. A mobilidade, a biodisponibilidade e a toxidez do As no ambiente solo é grandemente afetada pela natureza dos componentes mineralógicos da fração argila, pelo $\mathrm{pH}$ e pela presença de ânions fosfato (VIOLANTE e PIGNA, 2002). Assim como fosfato, o arsenato sofre adsorção química em óxidos de $\mathrm{Fe}$ e $\mathrm{Al}$, aluminossilicatos amorfos e, em pequena extensão, em argilossilicatos. $\mathrm{O}$ arsenato é o ânion do ácido forte, $\mathrm{H}_{3} \mathrm{AsO}_{4}$, que possui três valores de $\mathrm{pKa}(2,24$, 6,94 e 11,5), sendo adsorvido com maior eficiência em valores baixos de pH (McBRIDE, 1994), ou seja, possui baixa mobilidade em solos ácidos com alto conteúdo de argila ou óxidos. O coeficiente de distribuição $(\mathrm{Kd})$ de As na presença de outros ânions 

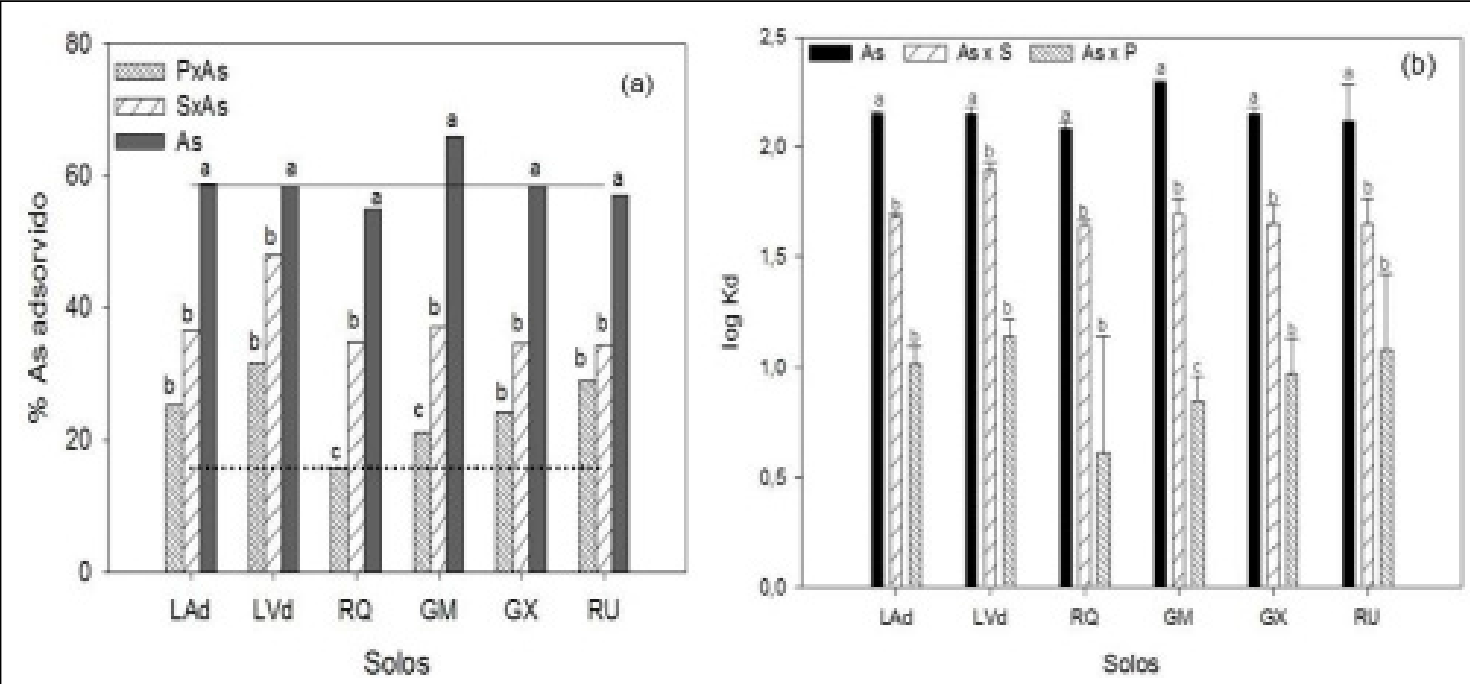

Figura 1 - Porcentagem de arsênio adsorvido (a) e log Kd (b)na ausência e presença de sulfato (S xAs) e fosfato (P x As). Adsorção conduzida em força iônica $15 \mathrm{mmol} \mathrm{L}^{-1}$, em pH 5,5 e em quantidades equimolares dos ânions sulfato, fosfato e arsenato Letras minúsculas comparam porcentagem adsorvida de arsenato na ausência e na presença do fosfato e sulfato, sendo que as médias seguidas de mesma letra não diferem entre si pelo teste de Scott-Knott a 5\% de probabilidade.

é ferramenta importante na predição do risco de mobilização do contaminante para horizontes mais profundos, principalmente em áreas contaminadas com As e que recebam fertilizantes.

Os resultados obtidos para adsorção competitiva entre arsenato/sulfato e arsenato/ fosfato, nos seis solos-alvo deste estudo, apontam para a possibilidade do aumento da disponibilidade do arsenato quando os solos são adubados com fertilizantes que contenham os oxiânions sulfato ou fosfato. A aplicação de fertilizantes fosfatados ou sulfatados pode ser utilizada como promotores do aumento do arsenato na solução do solo em programas de fitorremediação, especialmente, quando do uso da fitoextração (SALT et al., 1995). Entretanto, a diminuição da adsorção do arsenato na presença do sulfato e fosfato pode conduzir ao aumento da biodisponibilidade do arsenato e à entrada deste na cadeia alimentar.

\section{CONCLUSÃO}

Os solos Neossolo Flúvico (RU), Gleissolo Háplico (GX), Gleissolo Melânico (GM), Latossolo Vermelho Distrófico (LVd), Neossolo Quartzarênico (RQ) e o Latossolo Amarelo Distrófico (LAd) apresentaram teores médios semitotais de As entre $0,14 \pm 0,1 \mathrm{mg} \mathrm{kg}^{-1}$ (Gleissolo Háplico -GX) e 9,3 $\pm 1,0 \mathrm{mg} \mathrm{kg}^{-1}$ (Latossolo Vermelho Distrófico LVd), teores esses normalmente encontrados em solos ditos não contaminados $\left(<10 \mathrm{mg} \mathrm{kg}^{-1}\right)$.
O solo Gleissolo Melânico (GM) apresentou porcentagem de arsênio adsorvida, na ausência de outros oxiânions, superior aos demais solos. A porcentagem de As adsorvida correlacionouse significativamente com a relação $\mathrm{Fe}_{\mathrm{ox}} / \mathrm{Fe}_{\mathrm{DCB}} \mathrm{A}$ adição de fosfato e sulfato diminuiu a adsorção de arsenato nos seis solos estudados, aumentando assim sua concentração na solução do solo, ou seja, sua disponibilidade.

\section{AGRADECIMENTOS}

financeiro

Os autores agradecem a FAPEMIG pelo suporte

\section{REFERÊNCIAS}

ALLOWAY, B.J. Heavy metals in soil. New York: John Wiley e Sons, 1990. 339p.

AMERICAN PUBLIC HEALTH ASSOCIATION (APHA) Standard methods for the examination of water and wastewater. 17.ed. Washington, 1989. 1644p.

BAIRD, C. Química ambiental. 2.ed. Porto Alegre: Bookman, 2002. 621p.

BASU, A. et al. Genetic toxicology of a paradoxical human carcinogen, arsenic: a review. Mutation Research, v.488, p.171194, 2001. Disponível em: <http://www.sciencedirect.com/ science/article/pii/S1383574201000564>. Acesso: jun. 2009. doi: 10.1016/S1383-5742(01)00056-4.

CAO, X.; MA, L.Q. Effects of compost and phosphate amendments on arsenic mobility in soils and arsenic uptake by the hyperaccumulator, Pteris vittata L. Environmental

Ciência Rural, v.43, n.6, jun, 2013. 
Pollution, v.126, p.157-167, 2003. Disponível em: <http://www. sciencedirect.com/science/article/pii/S0269749103002082> Acesso: jun. 2009. doi:10.1016/S0269-7491(03)00208-2.

CAMPOS, M.L. et al. Teor e capacidade máxima de adsorção de arsênio em Latossolos brasileiros. Revista Brasileira de Ciência do Solo, v.31, p.1311-1318, 2007. Disponível em: <http://www. scielo.br/pdf/rbcs/v31n6/10.pdf>. Acesso: jul. 2009. doi: 10.1590/ S0100-06832007000600010.

CAMPOS, M.L. et al. Força iônica da solução de equilíbrio na adsorção de arsênio em latossolos brasileiros. Pesquisa Agropecuaria brasileira, v.41, n.3, p.457-460, 2006. Disponível em: <http://www.scielo.br/pdf/pab/v41n3/29117.pdf>. Acesso: jul. 2009. doi: 10.1590/S0100-204X2006000300012.

CHARLET, L. et al. Sulfate adsorption on a variable charge soil and on reference minerals. Agriculture, Ecosystems and Environment, v.47, p.87-102, 1993. Disponível em: <http:// www.sciencedirect.com/science/article/pii/016788099390104W>. Acesso: ago. 2009. doi:10.1016/0167-8809(93)90104-W.

CURI, N.; FRANZMEIER, D.P. Effect of parent rocks on chemical and mineraligical properties of some Oxisols in Brasil. Soil Science Society of America Journal, v.51, p.153-158, 1987. Disponível em: <https://www.soils.org/publications/sssaj/ pdfs/51/1/SS0510010153>. Acesso em: jan. 2009. doi:10.2136/ss saj1987.03615995005100010033x.

FERNANDES, L.A. et al. Frações de fósforo e atividade da fosfatase ácida em plantas de feijoeiro cultivadas em solos de várzea. Revista Brasileira de Ciência do Solo, v.24, p.561-571, 2000.

FERREIRA, D.F. Manual do sistema Sisvar para análises estatísticas. Lavras: UFLA, 2000. 63p.

FITZ, W.J.; WENZEL, W.W. Arsenic transformations in soilrhizosphere-plant system: Fundamentals and potential application to phytoremediation. Journal of Biotechnology, v.99, p.259-278, 2002. Disponível em: <http://www.sciencedirect.com/science/ article/pii/S0168165602002183 > . Acesso: dez. 2008. doi:10.1016/ S0168-1656(02)00218-3.

GEELHOED, J.S. et al. Phosphate and sulfate adsorption on goethite: single anion and competitive adsorption. Geochimica et CosmochimicaActa, v.61,p.2389-2396, 1997. Disponível em: $<$ http:// www.sciencedirect.com/science/article/pii/S0016703797000963> Acesso: dez. 2008. doi:10.1016/S0016-7037(97)00096-3.

GUSTAFSSON, J.P. Modeling competitive anion adsorption on oxide minerals and an allophane-containing soil. European Journal of Soil Science, v.52, p.639-654, 2001. Disponível em: <http://onlinelibrary. wiley.com/doi/10.1046/j.1365-2389.2001.00414.x/abstract;jsessionid= FEA47FF713EE0B5B584C9C3FEE8678FC.d04t02>. Acesso: dez. 2008. doi:10.1046/j.1365-2389.2001.00414.x

HIEMSTRA T.; VAN RIEMSDIJK W.H. Surface structural ion adsorption modeling of competitive binding of oxyanions by metal (hydr)oxides. Journal of Colloid and Interface Science, v.210, p.182-193, 1999. Disponível em: <http://www.sciencedirect.com/ science/article/pii/S0021979798959045>. Acesso: ago. 2009. doi: $10.1006 /$ jcis. 1998.5904

JAIN, A. et al. Arsenite and arsenate adsorption on ferrihydrite: Surface charge reduction and net $\mathrm{OH}^{-}$release stoichiometry.
Environmental Science and Technology, v.33, p.11791184, 1999. Disponível em: <http://lib3.dss.go.th/fulltext/ Journal/Environ\%20Sci.\%20Technology1998-2001/1999/ no.8/8,1999\%20vol.33,no8,p.1179-1184.pdf>. Acesso: dez. 2008. doi:10.1021/es980722e

MAGALHÃES, V.F. et al. Arsenic contamination and dispersion in the Engenho Inlet, Sepetiba Bay, SE, Brazil. Water, Air, and Soil Pollution, v.129, p.83-90, 2001.

MAGALHÃES, V.F.; PFEIFFER, W.C. Arsenic concentration in sediments near a metallurgical plant (Sepetiba Bay, Rio de Janeiro, Brazil). Journal of Geochemical Exploration, v.52, p.175-181, 1995. Disponível em: <http://www.sciencedirect.com/ science/article/pii/0375674294000257>. Acesso: dez. 2008. doi: 10.1016/0375-6742(94)00025-7.

MATSCHULLAT, J. Arsenic in the geosphere: a review. Science of the Total Environmental, v.249, p.297-312, 2000. Disponível em: < http://www.sciencedirect.com/science/article/pii/ S0048969799005240>. Acesso: dez. 2008. doi: 10.1016/S00489697(99)00524-0.

MCBRIDE, M.B. Environmental chemistry of soils. New York: Oxford University, 1994. 406p.

MELAMED, R.; JURINAK, J. J.; DUDLEY, L. M. Effect of adsorbed phosphate on transport of arsenate through an Oxisol. 1995. Soil Science Society American Journal, v. 59, 12891294.Disponível em: <https://www.crops.org/publications/sssaj/ abstracts/59/5/SS0590051289>. Acesso: Julho de 2009. doi: 10.2136/sssaj1995.03615995005900050012x.

NIEDZIELSKI, P.; SIEPAK, M.; SIEPAK, J. Comparison of modifiers for determination of arsenic, antimony and selenium by atomic absorption spectrometry with atomization in graphite tube or hydride generation and in-situ preconcentration in graphite tube. 2002. Microchemical Journal, v. 72, 137-145. Disponível em: <http://www.sciencedirect.com/science/article/pii/ S0026265X01001618>. Acesso: Dezembro de 2008. doi:10.1016/ S0026-265X(01)00161-8

NRIAGU, J. O.; PACYNA, J. M. Quantitative assessment of worldwide contamination of air, water and soils with trace metals. 1988. Nature, v. 333, 134-139.Disponível em: <www.nature.com/ nature/journal/v333/n6169/abs/333134a0.html>. Acesso: julho 2009. doi: $10.1038 / 333134 \mathrm{a} 0$.

RAVEN, P. K.; JAIN, A.; LOEPPERT, R. H. Arsenite and arsenate adsorption on ferrihydrite: Kinetics, equilibrium, and adsorption envelopes. 1998. Environmental Science and Technology, v. 32, 344-349. Disponível em: <http://lib3.dss.go.th/fulltext/ Journal/Environ\%20Sci.\%20Technology1998-2001/1998/ no.3/3,1998\%20vol.32,no3,p344-349.pdf>. Acesso: jan. 2008. doi:10.1021/es970421p

ROYCHOWDHURY, T. et al. Arsenic and other heavy metals in soils from an arsenic-affected area of West Bengal, India. Chemosphere, v.49, p.605-618, 2002. Disponível em: $<$ http:// www.sciencedirect.com/science/article/pii/S0045653502003090>. Acesso: jul. 2009. doi: 10.1016/S0045-6535(02)00309-0.

SALT, D.E. et al. Mechanism of cadmium mobility and accumulaton in Indian mustard. Plant Physiology, v.109, p.14271433, 1995. Disponível em: <http://www.ncbi.nlm.nih.gov/pmc/ articles/PMC157678/pdf/1091427.pdf>. Acesso: ago. 2009. 
SHEPPARD, S.C. Summary of phytotoxic levels of soil arsenic. Water, Air, and Soil Pollution, v.64, p.539-550, 1992. Disponível em: $<$ http://www.ijest.org/?_action=articleInfo\&article $=505>$. Acesso: jul. 2009. doi: $10.1007 / \mathrm{BF} 00483364$.

SKOOG, D.A. Princípios de análise instrumental. 5.ed. Porto Alegre: Bookman, 2002. 836p.

SMEDLEY, P.L.; KINNIBURGH, D.G. A review of the source, behaviour and distribution of arsenic in natural waters. Applied Geochemistry, v.17, p.517-568, 2002. Disponível em: <http:// www.sciencedirect.com/science/article/pii/S0883292702000185>. Acesso: nov. 2009. doi:10.1016/S0883-2927(02)00018-5.

SMITH, E. et al. Arsenic in the soil environment: a review. Advances in Agronomy, v.64, p.149-195, 1998.

SMITH, E. et al. Chemistry of arsenic in soils: I. Sorption of arsenate and arsenite by four Australian soils. Journal of Environmental Quality, v.28, p.1719-1726, 1999. Disponível em: <https://www. soils.org/publications/jeq/abstracts/28/6/JEQ0280061719>. Acesso: nov. 2008. doi:10.2134/jeq1999.00472425002800060005x.

SOUZA, R.F. et al. Calagem e adubação orgânica: Influência na adsorção de fósforo em solos. Revista Brasileira de Ciência do Solo, v.30, p.975-983, 2006. Disponível em: <http://www. scielo.br/pdf/rbcs/v30n6/a07v30n6.pdf>. Acesso: jul. 2009. doi: 10.1590/S0100-06832006000600007.

SPARKS, D.L. Environmental soil chemistry. California: Academic, 1995. 267p.
USEPA (UNITED STATES ENVIRONMENTAL PROTECTION AGENCY). Test methods for evaluating solid waste, physical/ chemical methods. SW-846. 3.ed. Washinton: Government Printing Office, 1995. 293p.

TAN, K.H. Principles of soil chemistry. 2.ed. New York: Marcel Dekker, 1992. 362p.

WENZEL, W.W. et al. Arsenic in field-collected soil solution and extracts of contaminated soils and its implication to soil standards. Journal Plant Nutrition Soil Science, v.165, p.221-228, 2002. Disponível em: <http://onlinelibrary.wiley.com/doi/10.1002/15222624(200204)165:2\%3C221::AID-JPLN221\%3E3.0.CO;2-0/pdf $>$. Acesso: nov. 2008. doi:10.1002/1522-2624(200204)165:2<221::AIDJPLN221>3.0.CO;2-0.

VIOLANTE, A.; PIGNA, M. Competitive sorption of arsenate and phosphate on different clay minerals and soils. Soil Science Society of America Journal, v.66, p.1788-1796, 2002. Disponível em: <https://www.soils.org/publications/sssaj/articles/66/6/1788>. Acesso: maio, 2009. doi: 10.2136/sssaj2002.1788.

ZAKHAROVA, T. et al. Health cancer risk assessment for arsenic exposure in potentially contaminated areas by plants fertilizer: a possible regulatory approach applied to a case study in Moscow Region-Russia. Regulatory Toxicology and Pharmacology, v.36, p.22-23, 2002. Disponível em: <http://www.sciencedirect. com/science/article/pii/S0273230002915618>. Acesso: ago. 2009 doi:10.1006/rtph.2002.1561.

Ciência Rural, v.43, n.6, jun, 2013. 